\title{
Investigação da variação de pressão em sistemas de vazão lateral através do princípio de Bernoulli
}

Investigation of the pressure variation in side flow systems through the Bernoulli principle

\author{
E. C. B. Moraes*10, H. C. Baptista² ${ }^{2}$ A. B. De $\operatorname{Souza}^{1}$, L. R. Dos Santos ${ }^{2}$ \\ ${ }^{1}$ Instituto Federal de Educação, Ciência e Tecnologia, Coordenação de Ensino Médio, Tucuruí, PA, Brasil \\ ${ }^{2}$ Instituto Federal de Educação, Ciência e Tecnologia, Curso de Eletrotécnica, Tucuruí, PA, Brasil \\ ${ }^{3}$ Instituto Federal de Educação, Ciência e Tecnologia, Curso de Engenharia Sanitária e Ambiental, Tucuruí, PA, Brasil
}

Recebido em 13 de outubro de 2019. Revisado em 01 de novembro de 2019. Aceito em 11 de novembro de 2019

Especificamente, investigamos a variação de pressão ocorrido em sistemas simples de vazão lateral através do princípio de Bernoulli. Tal sistema de fluido é definido por um escoamento interno e externo a um recipiente de área de secção transversal constante, através de um orifício localizado em sua lateral. O escoamento pode ser controlado ou não, de forma a garantir ou não a aceleração constante no sistema. Ressaltamos que reproduzimos tais sistemas devido a ação do campo gravitacional. Os resultados mostram que o conhecimento da viscosidade dos fluidos são imprescindíveis na interpretação da variação de pressão do sistema.

Palavras-chave: Sistema de vazão lateral, Variação de pressão, Altura da Quantidade Dinâmica de Fluido e Alcance de Escoamento.

Specifically, we investigated the pressure variation that occurred in simple lateral flow systems through the Bernoulli principle. Such a fluid system is defined by an internal and external flow to a container of constant crosssectional area through a hole located on its side. Flow may or may not be controlled to ensure constant acceleration in the system. We emphasize that we reproduce the referent system due to the action of the gravitational field. The results show that the knowledge of the fluid viscosity is essential in the interpretation of the system pressure variation.

Keywords: Flow Side System, Pressure Variation, Height of the Quantity of Fluid Dynamic and Flow of Reach.

\section{Introdução}

Atualmente, obter e analisar o comportamento da variação de pressão de sistemas de fluidos é obter informações sobre a sua direção de deslocamento, campo de velocidade, energia mecânica total e entre outras grandezas físicas. Tais grandezas permitem compreender, por exemplo, a planagem de um avião, de um pássaro, o movimento de animais marinhos, formação de furacões, maremotos, pequenas enchentes e entre outros fenômenos que podem ser explicados pela variação de pressão formada no local. A maioria desses exemplos podem ser definidos como sistemas de fluidos em equilíbrio e desequilíbrio dinâmico. Neste material, há resultados sobre a variação de pressão responsável pelo escoamento interno de fluidos na vertical, pelo recipiente de base e faces laterais retangulares, ejetados por um orifício circular em sua lateral, na forma de lançamento horizontal. Desse sistema, investigamos também o comportamento da altura da coluna de fluido para cada valor de alcance. Resultados mostram que a variação de pressão do referente sistema, investigado através da equação de Bernoulli [1], depende da viscosidade do fluido.

*Endereço de correspondência: elton.moraes@ifpa.edu.br

\section{Fundamentação teórica}

A variação de pressão em qualquer sistema de fluido é somente observado nos sistemas em desequilíbrio, contrariando o princípio de Pascal [2] Neste trabalho, investigamos a forma mais simples de um sistema de vazão lateral, onde o escoamento é devido a ação direta do campo gravitacional, promovendo escoamento interno na vertical e escoamento externo na forma de lançamento horizontal [3], como mostra a Figura 1$]$

Sobre o referente sistema, aplicamos o Princípio de Bernoulli na tentativa de expressar o comportamento da variação de pressão, desconsiderando a perda de energia na forma de calor e admitindo que o fluido é do tipo imcompressível 44. Também admitimos que há velocidade nos dois pontos de referência 1 e 2 (Figura 1). Desta forma, a equação de Bernoulli torna-se:

$$
\frac{P_{1}}{\mu \cdot g}+\frac{v_{1}^{2}}{2 \cdot g}+h_{1}=\frac{P_{2}}{\mu \cdot g}+\frac{v_{2}^{2}}{2 \cdot g}+h_{2},
$$

sendo $\mu$ a densidade do fluido, $g$ o valor da gravidade local, $v_{1}$ e $v_{2}$ as velocidades de escoamento na região 1 e 2 respectivamente. Para garantir que a variação de pressão 


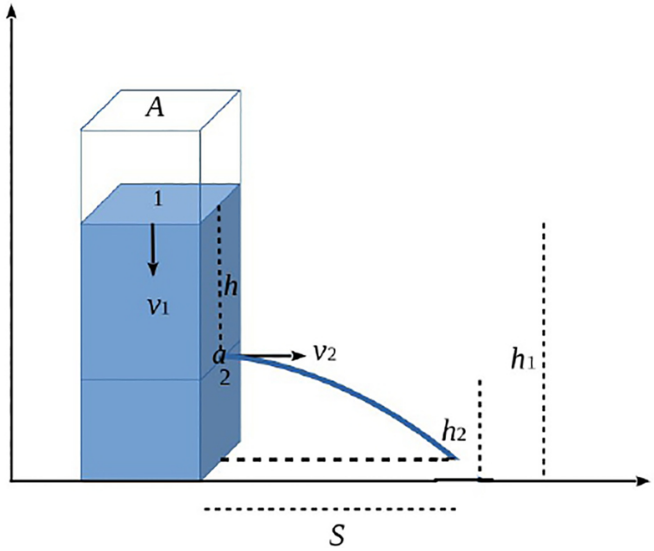

Figura 1: Sistema de Vazão Lateral.

deste sistema seja sempre positiva definimos $\Delta P=P_{2}-$ $P_{1}$. Sabendo que $h_{1}-h_{2}=h$, temos:

$$
\begin{gathered}
\frac{P_{2}-P_{1}}{\mu . g}=\left(h_{1}-h_{2}\right)-\left(\frac{v_{2}^{2}-v_{1}^{2}}{2 . g}\right) ; \\
\Delta P=\mu . g . h-\mu .\left(\frac{v_{2}^{2}-v_{1}^{2}}{2}\right) .
\end{gathered}
$$

Após muitas observações, verificamos que os valores do alcance de escoamento $S$ não se alteram quando obtidos sobre escoamento contínuo ou descontínuo, permitindo assim, a utilização da equação da continuidade [4], resultando em:

$$
v_{1}=\frac{a \cdot v_{2}}{A},
$$

sendo $a$ e $A$ a área do orifício circular lateral e a área da secção transversal de escoamento interno respectivamente. Considerando que o alcance de escoamento não depende da resistência do ar, temos:

$$
S=v_{2} . t
$$

que é justamente uma das equações do lançamento horizontal de corpos rígidos, em que $t=\sqrt{\frac{2 . h_{2}}{g}}$ representa o tempo de queda do ponto 2 até a base do recipiente. Desta forma:

$$
v_{2}=S \cdot \sqrt{\frac{g}{2 \cdot h_{2}}} .
$$

Substituindo a equação (6) na equação (4), temos:

$$
v_{1}=S \cdot \frac{a}{A} \cdot \sqrt{\frac{g}{2 . h_{2}}} .
$$

Substituindo as equações (6) e (7) na equação (3), concluimos que:

$\Delta P=\mu . g . h+$

$$
-\mu .\left(\frac{\left(S \cdot \sqrt{\frac{g}{2 \cdot h_{2}}}\right)^{2}-\left(S \cdot \frac{a}{A} \cdot \sqrt{\frac{g}{2 \cdot h_{2}}}\right)^{2}}{2}\right) ;
$$

$$
\Delta P=\mu \cdot g \cdot h-\frac{\mu \cdot g \cdot S^{2}}{4 \cdot h_{2}}\left(1-\frac{a^{2}}{A^{2}}\right) .
$$

Sendo assim, temos que $\Delta P=\Delta P(h, S)$, onde $h$ é a altura da quantidade dinâmica e $h_{2}$ é a altura da quantidade estática de fluido no sistema.

\section{Materiais e Métodos}

Para reprodução dos sistemas de fluidos utilizamos um equipamento de vidro, com área de secção transversal uniforme e retangular, com orifício na lateral. De fato, a vazão lateral ocorre quando desobstruimos o orifício do equipamento contendo o fluido. Os fluidos utilizados foram água comum, óleo ABC (óleo de soja ) e óleo Q (óleo queimado de motor de moto) de densidade $10^{-3} \mathrm{~kg} / \mathrm{cm}^{3}$, $8,93.10^{-4} \mathrm{~kg} / \mathrm{cm}^{3}$ e $8,45.10^{-4} \mathrm{~kg} / \mathrm{cm}^{3}$ respectivamente. Sem necessitar de medidas, verifica-se que o óleo Q é muito mais viscoso que óleo $\mathrm{ABC}$ e ambos possuem viscosidade maiores que a da água.

Antes de determinar a variação de pressão, primeiramente obtemos os valores de altura da quantidade dinâmica de fluido a cada valor fixo de alcance de escoamento. O alcance máximo fixado foi de $S_{m}=25 \mathrm{~cm}$ e as demarcações foram sobre uma fita métrica ao passo de $-2 \mathrm{~cm}$ até $5 \mathrm{~cm}$ de alcance.

Após determinar os valores da altura da quantidade dinâmica de fluido em valores fixos de alcance, obtivemos os valores da variação de pressão através de um programa computacional chamado Qtiplot. Para o desenvolvimento dos cálculos, fixamos $h_{2}=13,5 \mathrm{~cm}$ e consideramos $g=980 \mathrm{~cm} / \mathrm{s}^{2}$ e $a^{2} / A^{2}=0$, pois, $a$ e $A$ são iguais a $0,283 \mathrm{~cm}^{2}$ de raio $r=0,3 \mathrm{~cm}$ e $120,96 \mathrm{~cm}^{2}$ de dimensões $11,2 \mathrm{~cm} X 10,8 \mathrm{~cm}$ respectivamente, ou seja, $a$ é muito menor que $A$, neste caso.

\section{Resultados e Discussões}

Os resultados mostram que a função que descreve a dependência da altura da quantidade dinâmica de fluido para cada valor de alcance é semelhante a uma equação do $2^{\mathrm{o}}$ grau e que sua taxa de crescimento é tanto maior quanto maior for a viscosidade do fluido investigado 5,6 . Para cada fluido, foram realizadas três medidas da altura de sua quantidade dinâmica, distribuídas nas tabelas correspondentes as Figuras $2,3 \mathrm{e} 4$

\begin{tabular}{|c|c|c|c|c|}
\cline { 2 - 5 } \multicolumn{1}{c|}{} & $\mathrm{S}[\mathrm{X}]$ & $\mathrm{h} 1[\mathrm{Y}]$ & $\mathrm{h} 2[\mathrm{Y}]$ & $\mathrm{h} 3[\mathrm{Y}]$ \\
\hline 1 & 25 & 14.2 & 13.5 & 13.7 \\
\hline 2 & 23 & 12.6 & 12 & 11.4 \\
\hline 3 & 21 & 10.1 & 10.9 & 9.8 \\
\hline 4 & 19 & 8.9 & 8.4 & 8.1 \\
\hline 5 & 17 & 7.4 & 7 & 6.5 \\
\hline 6 & 15 & 5.9 & 5.6 & 5.4 \\
\hline 7 & 13 & 4.6 & 4.5 & 4.5 \\
\hline 8 & 11 & 3.6 & 3.6 & 3.4 \\
\hline 9 & 9 & 2.6 & 2.6 & 2.6 \\
\hline 10 & 7 & 2.1 & 2 & 2 \\
\hline 11 & 5 & 1.7 & 1.6 & 1.5 \\
\hline
\end{tabular}

Figura 2: Distribuição de três medidas da altura da quantidade dinâmica de água em valores fixos do alcance. 


\begin{tabular}{|c|c|c|c|c|}
\cline { 2 - 5 } & $\mathrm{S}[\mathrm{X}]$ & $\mathrm{h} 1[\mathrm{Y}]$ & $\mathrm{h} 2[\mathrm{Y}]$ & $\mathrm{h} 3[\mathrm{Y}]$ \\
\hline $\mathbf{1}$ & 25 & 22 & 22.1 & 22.1 \\
\hline 2 & 23 & 19.4 & 19.5 & 19.1 \\
\hline 3 & 21 & 16.6 & 16.6 & 16.6 \\
\hline 4 & 19 & 14.4 & 14.1 & 14.1 \\
\hline 5 & 17 & 12 & 11.9 & 11.9 \\
\hline 6 & 15 & 10 & 10.1 & 10 \\
\hline 7 & 13 & 8.5 & 8.3 & 8.3 \\
\hline 8 & 11 & 6.9 & 6.7 & 6.9 \\
\hline 9 & 9 & 5.4 & 5.4 & 5.4 \\
\hline 10 & 7 & 4.4 & 4.2 & 4.5 \\
\hline 11 & 5 & 3.4 & 3.2 & 3.3 \\
\hline
\end{tabular}

Figura 3: Distribuição de três medidas da altura da quantidade dinâmica de óleo $A B C$ em valores fixos do alcance.

\begin{tabular}{|c|c|c|c|c|}
\cline { 2 - 5 } \multicolumn{1}{c|}{} & S $[\mathrm{X}]$ & $\mathrm{h} 1[\mathrm{Y}]$ & $\mathrm{h} 2[\mathrm{Y}]$ & $\mathrm{h} 3[\mathrm{Y}]$ \\
\hline 1 & 25 & 28.2 & 28.6 & 28.5 \\
\hline 2 & 23 & 24.8 & 25.3 & 25.1 \\
\hline 3 & 21 & 22 & 22.6 & 21.9 \\
\hline 4 & 19 & 19.2 & 19.6 & 18.9 \\
\hline 5 & 17 & 16.1 & 16.8 & 16.4 \\
\hline 6 & 15 & 13.4 & 14.1 & 13.8 \\
\hline 7 & 13 & 11.2 & 11.5 & 11.3 \\
\hline 8 & 11 & 9 & 9.4 & 9.3 \\
\hline 9 & 9 & 7.2 & 7.5 & 7 \\
\hline 10 & 7 & 5.6 & 5.6 & 5.5 \\
\hline 11 & 5 & 4.4 & 4.4 & 4.4 \\
\hline
\end{tabular}

Figura 4: Distribuição de três medidas da altura da quantidade dinâmica de óleo $Q$ em valores fixos do alcance.

Para os valores da variação de pressão, consideramos os valores fixos de alcance e os valores médios da altura da quantidade dinâmica de cada fluido, distribuídos na tabela correspondente a Figura 5 gerando assim, os gráficos correspondentes as Figuras 6 e 7 .

\section{Conclusões}

Dos resultados, destacamos a forte influência da viscosidade dos fluidos envolvidos, pois, de acordo com tabela da Figura 5 para obtenção do alcance de $23 \mathrm{~cm}$ por exemplo, obtemos na média $12 \mathrm{~cm}, 19.34 \mathrm{~cm}$ e $25.06 \mathrm{~cm}$ de altura da quantidade dinâmica de água, óleo $\mathrm{ABC}$ e Óleo Q respectivamente. Ou seja, quanto maior a viscosidade do fluido maior deve ser a altura de sua quantidade dinâmica para o mesmo valor de alcance.

Diante dos resultados, como mostra a Figura 6 , percebemos que há uma proporcionalidade entre a altura da quantidade dinâmica de fluido com o quadrado do alcance e que a análise do coeficiente de viscosidade do fluido investigado deve ser o primeiro passo para uma possível obtenção de relações entre eles [7].

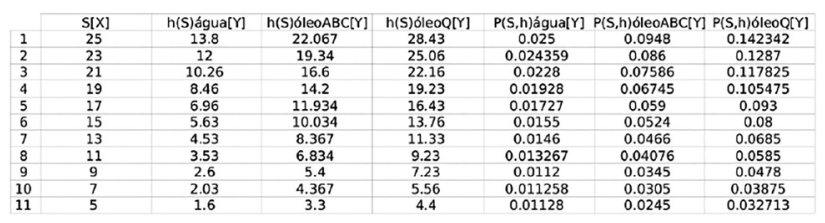

Figura 5: Distribuição dos valores da variação de pressão, através da média entre os valores da altura da quantidade dinâmica de fluido obtidas por valores fixos do alcance.

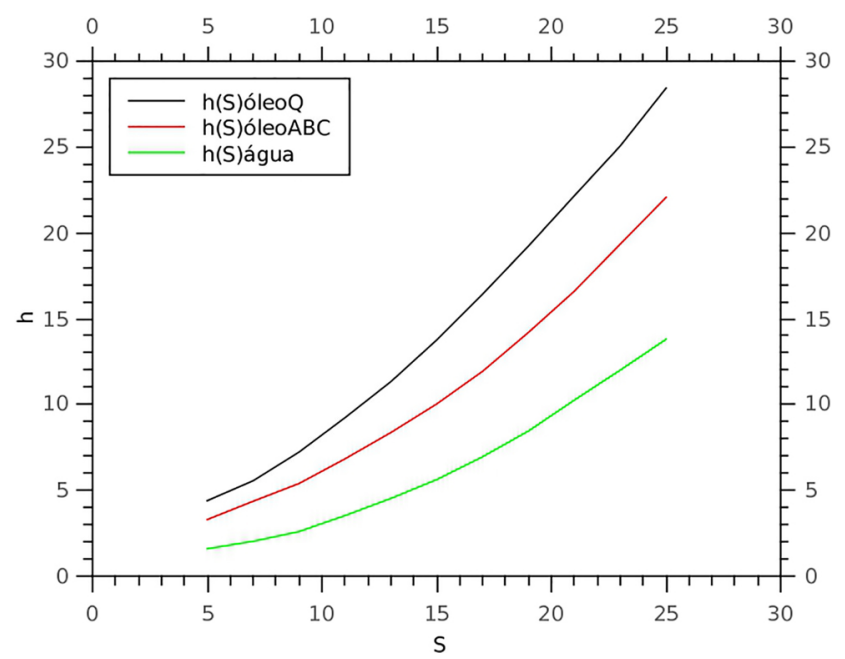

Figura 6: Gráfico da altura média da quantidade dinâmica de cada fluido sobre valores fixos de alcance.

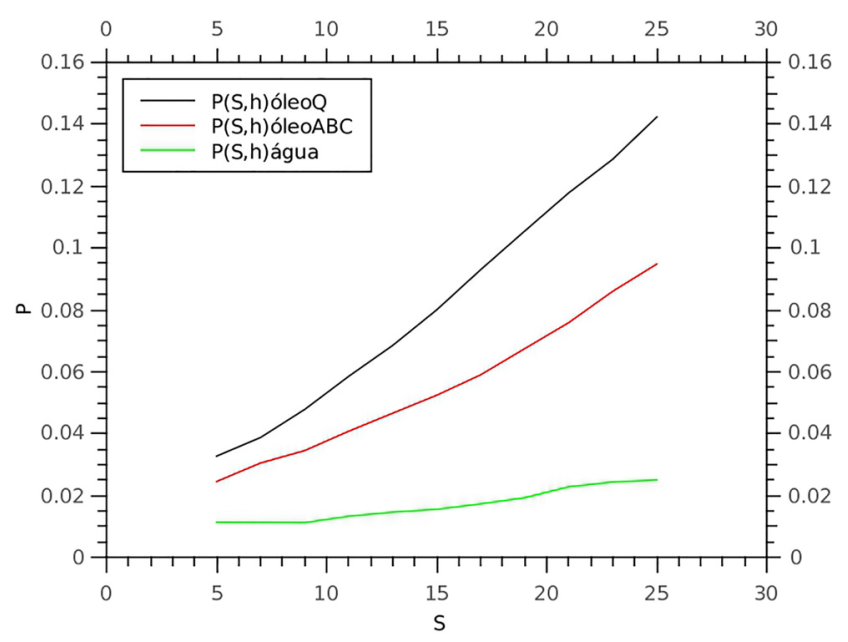

Figura 7: Gráfico da variação de pressão sobre a altura média da quantidade dinâmica de cada fluido, obtidos em valores fixos de alcance.

Concernente aos valores obtidos da variação de pressão do sistema, verificamos que, de fato, há uma queda de pressão devido o escoamento do fluido, porém, sua intensidade é tanto maior quanto maior for a viscosidade do fluido, como podemos observar no gráfico correspondente a Figura 7

\section{Referências}

[1] H.M. Nussenveig, Fluidos, Oscilações e Ondas, Calor 2: Curso de Física Básica (Editora Blucher, São Paulo, 2002), v.2.

[2] R. Resnick, D. Halliday e K.S. Krane, Física 2 (Editora LTC, Rio de Janeiro, 1960), v.2.

[3] R. Resnick, D. Halliday e K.S. Krane, Física 1. (Editora LTC, Rio de Janeiro, 1960), v.2.

[4] M.S. Cattani, Elementos de Mecânica dos Fluidos (Edgard Blucher: LTDA, São Paulo, 2005), v.1. 
[5] F.A. Pereira, Escoamento Laminar de Líquidos Não Newtonianos em Seções Anulares:Estudos de CFD e Abordagem Experimental. Tese de Doutorado, Universidade Federal De Uberlândia, Minas Gerais (2006).

[6] R.W. Fox e A.T. McDonald, Introdução À Mecânica dos Fluidos (Editora LTC, Rio de Janeiro, 2001), v.1.

[7] J.C.A. Marcedo, Determinação Experimental da Função que Modela o Escoamento de um Líquido. Dissertação de Mestrado, Universidade Federal de São Carlos, São Paulo (2010). 